\title{
A fast and simple bonding method for low cost microfluidic chip fabrication
}

\author{
Zhifu Yin ${ }^{*}$, Helin Zou ${ }^{* *}$
}

\begin{abstract}
With the development of the microstructure fabrication technique, microfluidic chips are widely used in biological and medical researchers. Future advances in their commercial applications depend on the mass bonding of microfluidic chip. In this study we are presenting a simple, low cost and fast way of bonding microfluidic chips at room temperature. The influence of the bonding pressure on the deformation of the microchannel and adhesive tape was analyzed by numerical simulation. By this method, the microfluidic chip can be fully sealed at low temperature and pressure without using any equipment. The dye water and gas leakage test indicated that the microfluidic chip can be bonded without leakage or block and its bonding strength can up to $0.84 \mathrm{MPa}$.
\end{abstract}

K e y w o r d s: microfluidic chip, tape assist bonding, numerical simulation

\section{Introduction}

Microfluidic chips can deal with the behavior, precise control and manipulation of fluids that are geometrically constrained to a typically sub-millimeter scale [1]. They have gained increasing attention in recent years due to their portability, minimized, high degree of integration, and little reagent consumption [2]. Microfluidic chips can be used in a number of fields, particularly in medical and biological fields.chanical strength, temperature and irradiation resistance and moisture resistance are other distinct advantages.

To fabricate a microfluidic chip, lots of methods can be utilized such as photolithograph [3], micromachining [4], wet etching [5].However, by those methods, microchannels were fabricated in glass, quartz or silicon substrate. The high cost and processing complicacy during fabrication limit the application of the microfluidic chips. Future advances in microfluidic chips and their utilization in commercial applications depends on the development and mass fabrication of low cost microfluidic chips. In the past decades, hot embossing [6], injection molding [7], and nanoimprinting [8] were developed. They are conceptually simple methods for patterning micro/nano resolution features on a polymer substrate. Polymers are less expensive and easier to be manipulated than glass or silicon substrates. They have become the most promising materials for fabricating microchannels with mass fabricating technologies. In contrast to those state-of-the-art fabrication methods, polymer fabricating methods show considerably better results in terms of cost, productivity, and simple operation in microfabrication procedures.

However, to fabricate microfluidic chips, the open microchannels need to be sealed. A number of different bonding techniques such as APTES bonding [9, 10], thermal bonding [11-13], solvent bonding [14, 15] and microwave welding methods [16] have been reported in recent several years. Among these bonding methods, thermal bonding has attracted increasing interests due to its simple and low-cost characters. However, the thermal bonding method does suffer from some disadvantages due to the reflow phenomenon of the polymer sheet [17]. During thermal bonding process, when the bonding temperature and pressure are not carefully controlled, the patterned microchannels will easily deformed and the cover plate will easily flow into the microchannels, causing partial or complete microchannels closure. A fast microchannel bonding method with low pressure and temperature need to be developed.

In this study we are presenting a new way of bonding microchannels at room temperature namely tape assist bonding method. It is to be used in the high-volume production of microfluidic chips without making the whole process too time-consuming and too costly. The deformation of the microchannel during thermal bonding and tape assist bonding was studied. The relationship between the shape of the microchannel and its deformation was analyzed. The optimal bonding parameters for thermal bonding and tape assist bonding methods were determined.

\section{Experiments}

In this work, $1 \mathrm{~mm}$ PMMA sheets were chosen as the substrate material. They were purchased from Gayle Inc. (Burghausen, Germany). The Tg (glass transition temperature) of PMMA is $125^{\circ} \mathrm{C}$. The $1 \mathrm{~mm}$ PMMA

* School of Mechanical Science and Engineering, Jilin University, Changchun 130012, China, yinzf@jlu.edu.cn, ** Key Laboratory for Micro/ Nano Technology and Systems of Liaoning Province, Dalian University of Technology, Dalian 116024, China 

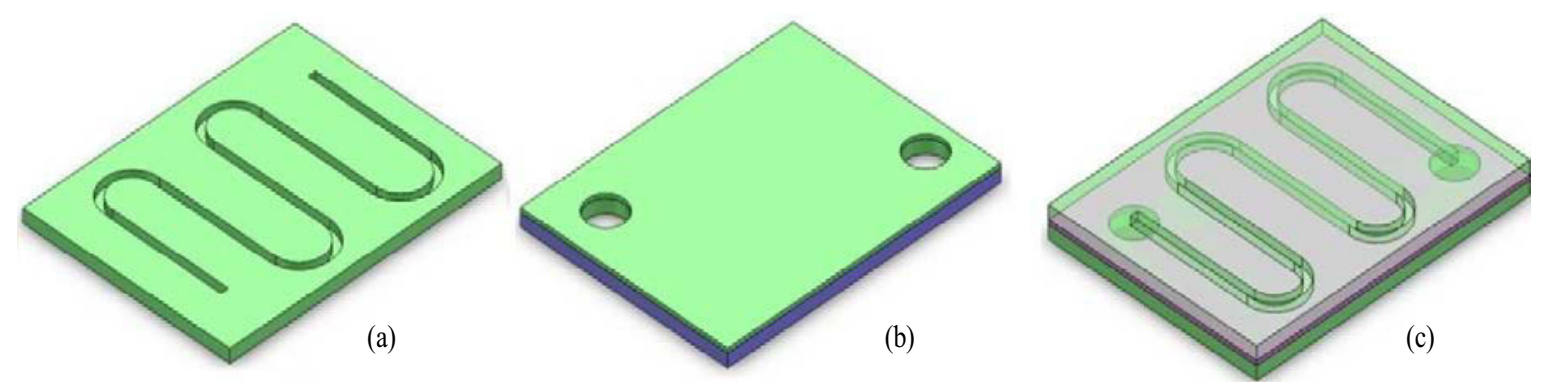

Fig. 1. Illustration of the bonding process for microfluidic chip fabrication
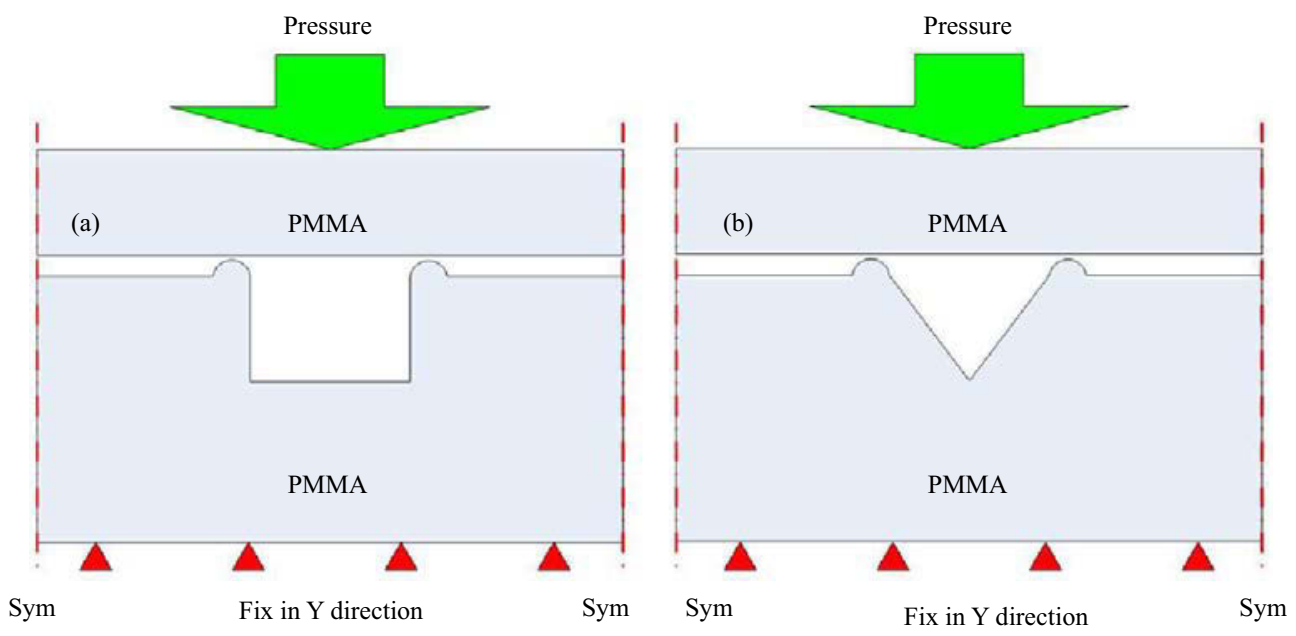

Fig. 2. The model used for numerical simulation of thermal bonding process

sheet was cut into $2 \mathrm{~cm} \times 3 \mathrm{~cm}$ square pieces. The prepared sample PMMA substrates were sonicated for 10 min in the cleaner (DZ-1, Jinan Xihua technologies Co., Ltd, Shandong, China), rinsed in de-ionized water and dried under a nitrogen stream. The microchannels were fabricated into the cleaned PMMA substrate as show in Fig. 1(a). Rectangular microchannels can be fabricated by hot embossing, while triangular microchannels can be fabricated by laser erosion.

To seal the microchannels at low pressure and temperature, tape assist bonding method was used. As shown in Fig. 1(b), double sides adhesive tape was pasted onto the cover plate. Before tape pasting, two holes were drilled in the cover plate and the tape. Then the substrate and cover plate were aligned under a custom-made microscope and bonded together by hand (with low bonding pressure). The PMMA microfluidic chip can be thus fabricated after bonding. Fig. 1(c).

\section{Results and discussion}

To seal the microchannels, there are a few of bonding method can be used, such as thermal bonding, solvent bonding, and microwave welding. Among them, thermal bonding is widely used due to its simple and low cost properties. However, the bonding process is time consuming, and the microchannels suffer from highly deformation if thermal bonding parameters are not carefully optimized. In this work, a tape assist bonding method was proposed. To compare those two methods, the deformation of the microchannels after bonding was compared by numerical simulation method. It is notable that the influencing conditions for those two methods are channelshape and bonding parameters.

\subsection{Thermal bonding method}

Rectangular and triangular microchannels are commonly used microstructures during polymer microfluidic chip fabrication, since those channels can be easily patterned into the polymer substrate by traditional microstructure fabrication technique. For example, rectangular microchannels can be fabricated by photolithography or wet etching method. Triangular microchannels can be fabricated by laser erosion method. To fabricate a microfluidic chip, the open microchannels should be sealed. For bonding the microchannels, thermal bonding method is usually employed. In this work, the deformation of those type microchannels during thermal bonding process was investigated.

The polymer substrate is always bonded at a temperature near Tg. For PMMA material, its $\mathrm{Tg}$ is 125 ${ }^{\circ} \mathrm{C}$. Near this temperature, polymer exhibits viscoelastic properties. The commonly used bonding temperature for PMMA is about $85^{\circ} \mathrm{C}[18,19]$, since at this temperature the microstructures can be easily boned without 


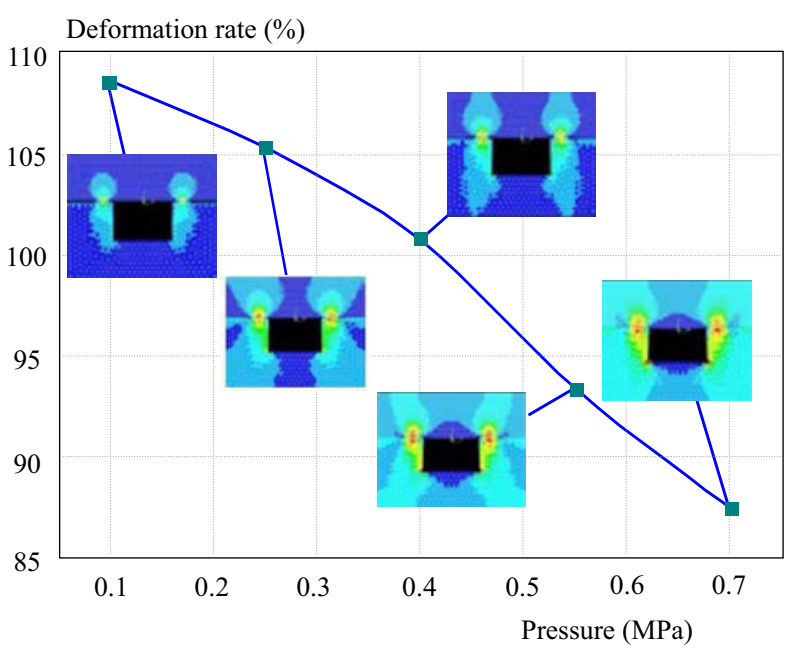

Fig. 3. The influence of the bonding pressure on the deformation of rectangular

highly deformation. Compared with the bonding time, bonding pressure can influence the deformation of the microchannels much more seriously. Thus we only study the influence of the bonding pressure on the deformation of the microchannels. To analyze the deformation of the microstructures during bonding process by numerical simulation, the viscoelastic model was employed. In this paper, generalized Maxwell model (a kind of viscoelastic model) was used to study the interrelationship between the bonding pressure and the deformation of the microchannels. The generalized Maxwell model can be written as [20, 21],

$$
Y(t)=E_{\infty}+\sum_{i=1}^{n} E_{i} e^{-\frac{t}{\tau_{i}}}
$$

where $E_{\infty}, E_{i}$ and $\tau_{i}$ are constants of the generalized Maxwell model. During hot embossing, the temperature dependence of the viscoelastic properties of the polymer can be expressed by using time-temperature conversion based on the Williams-Landel-Ferry (WLF) shift factors $\mathrm{T}[22]$,

$$
\begin{gathered}
Y(t, T)=Y\left(\frac{t}{\alpha_{T}}, T_{0}\right), \\
\log \left(\alpha_{T}\right)=\frac{-C_{1}\left(T-T_{0}\right)}{C_{2}+\left(T-T_{0}\right)},
\end{gathered}
$$

where $T_{0}$ is the reference temperature and $C_{1}$ and $C_{2}$ are the parameters of material. It can be assumed that $C_{1}=17.4$ and $C_{2}=51.6$, when $T_{0}$ is the glass transition temperature of the given viscoelastic material $[23,24]$.

For simplification, the two-dimensional plain-strain condition is assumed. Since the microchannels have periodical structures, only one periodical microfeature was modeled to the finite element domain. The PMMA cover plate and the PMMA substrate was modeled as a viscoelastic material. A nonlinear FEM software ANSYS (ANSYS inc., Pittsburgh, Pennsylvania, USA) was employed to solve the constitutive and geometric equation

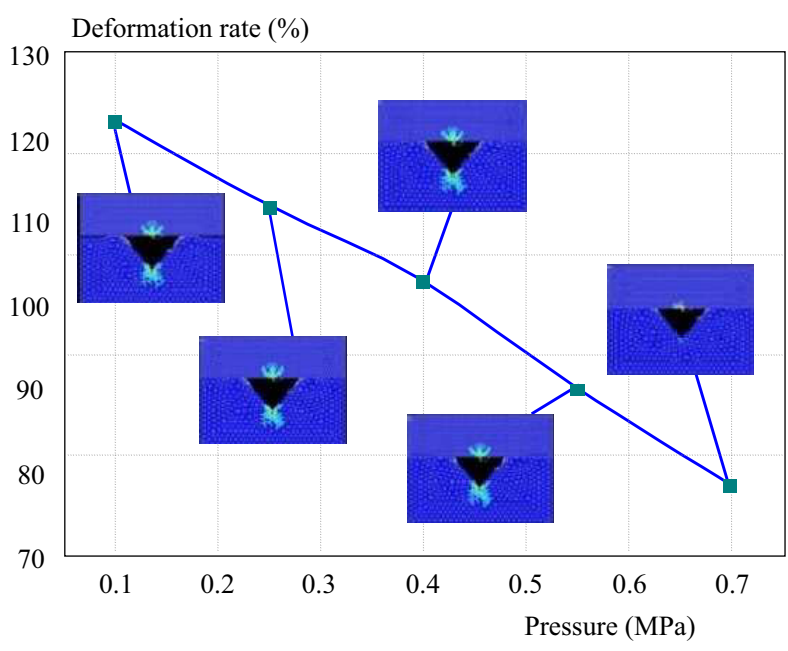

Fig. 4. The influence of the bonding pressure on the deformation of triangular microchannels (thermal bonding method)

simultaneously. Symmetric boundaries were applied on both sides of the cover plate and the substrate. The Yaxis displacement of the bottom surface of the substrate was zero due to the PMMA substrate supported by a fixed bonding board. The geometric model is shown in Fig. 2.

The following parameters: open circuit voltage, short circuit current, maximum power point, fill factor (FF) and efficiency can be determined. The results obtained experimentally from the measurements under illumination are illustrated in Fig. 3, where the fill factor dispersion for the both sample series with different fingers density is given. Of course, all samples show quite poor PV parameters when above mentioned optimization is missing. For the purpose of this paper, the light $I-V$ measurements were carried out only to compare the behavior of the prepared samples under light.

Figures 3 to 4 show the influence of the bonding pressure on the deformation of the microchannels by numerical simulation. To analyze the deformation of the microchannels, deformation rate is defined. It refers to the area of the cross-section of the microchannel before bonding to that after bonding. It indicates that the microchannel is not fully sealed when the deformation rate is larger than 100\%. From Fig. 3 and Fig. 4, One can see that the deformation rate decrease with the increase of the bonding pressure. For rectangular and triangular microchannel, the channel can be fully sealed without any leakage at bonding pressure of $0.55 \mathrm{MPa}$. At such high bonding pressure, the microchannel is highly deformed. The deformation rate drops to $93 \%$ and $90 \%$ for rectangular and triangular microchannel. To bond uneven substrate patterned with microchannel, thermal bonding method is not accessible due to the high deformation of the bonded microchannels. 


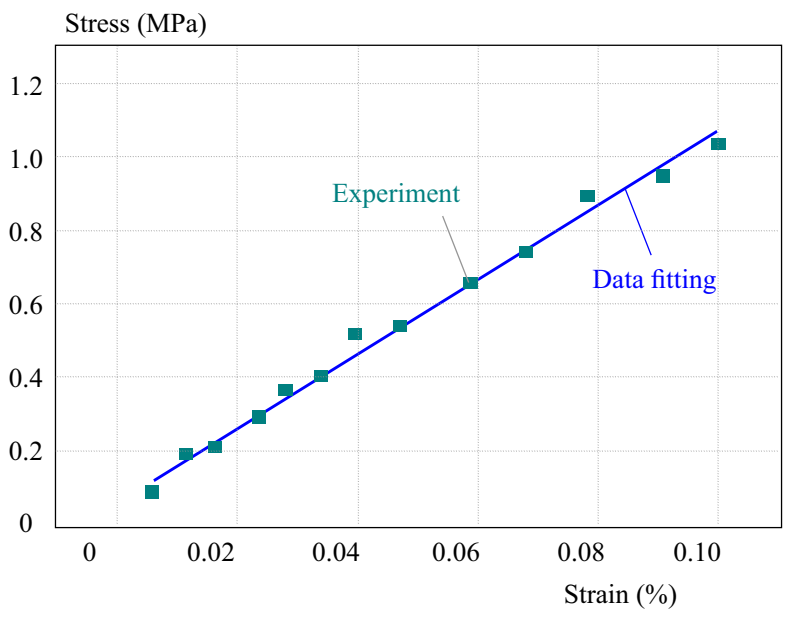

Fig. 5. The Young modulus of the adhesive tape is $10.1 \mathrm{MPa}$

\subsection{The Young modulus of the adhesive tape}

In the present work, tape assist bonding method was proposed for fully bonding the microchannels without serious deformation. To measure the Young modulus of the adhesive tape, compressing equipment was used. The equipment consists of a forcing system, a heating/cooling system and a monitoring/controlling system. During compressing process, the forcing system provides necessary compression force with an accuracy of $0.5 \mathrm{~N}$. The force and displacement can be dynamically controlled and recorded by the monitoring/ controlling system. Figure 5 shows the strain-stress curves of the adhesive tape. There is a nearly linear relationship between the strain and the stress. Fitted by the linear function in the Origin Pro 8 (OriginLab Corp. Northampton, USA), the Young modulus of the adhesive tape can be calculated. The average correlation of curve fitting at different mixing ratio is as high as $\mathrm{R} 2=98.1 \%$. The fitting results show that the Young modulus is $10.1 \mathrm{MPa}$ for adhesive tape.

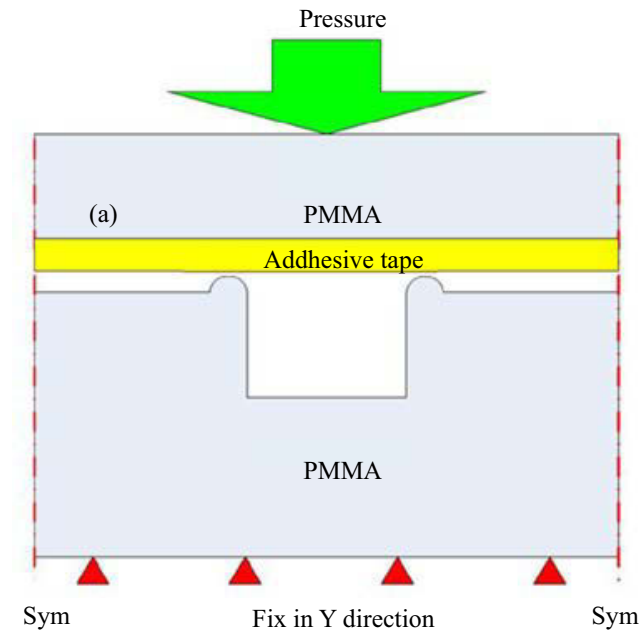

\subsection{Tape assist bonding method}

To fully bond the microchanels without serious microchannels deformation, a tape assist bonding method was proposed. This method can allow the microchannels bonded at room temperature without using any equipment. There are only two steps for this method. Firstly, adhesive tape is pasted onto the cover plate. Then the cover plate is bonded with the patterned substrate by hand. At room temperature, polymer displays elastic property. During numerical simulation, substrate and cover plate were considered to be elastic bodies. The geometric model is shown in Fig. 6. Adhesive tape exhibits hyperelastic property at room temperature. It can be assumed as an elastomer. We assume that adhesive tape is incompressible and isotropic. Its mechanical properties can be represented by Mooney-Rivlin model. The Mooney-Rivlin strain energy function is expressed as [25]

$$
W=C_{10}\left(I_{1-3}\right)+C_{01}\left(I_{2}-3\right),
$$

where, $C_{10}, C_{01}$ are physical constants characterizing the material, and $I_{1}, I_{2}$ are strain invariants.

As Hocheng reported [26], the physical constants $C_{10}$, $C_{01}$ can be evaluated by Youngs modulus of the polymer,

$$
C_{01}=0.25 C_{10}, \quad 6\left(C_{10}+C_{01}\right) \approx E
$$

where, $E$ is the Youngs modulus of the polymer.

Figure 7 and Fig. 8 show the influence of the bonding pressure on the deformation of rectangular and triangular microchannels by numerical simulation. It shows that at bonding pressure of $0.1 \mathrm{MPa}$ and $0.2 \mathrm{MPa}$ the microchannels cannot be fully bonded. When the bonding pressure increases to $0.3 \mathrm{MPa}$, the microchannels can be fully bonded. The deformation rates are $97 \%$ and $99 \%$ for rectangular and triangular microchannels. However, when the bonding pressure is larger than 0.3 MPa, the microchannels are deformed seriously. The deformation rates drop to $87 \%$ and $77 \%$ at bonding pressure of 0.5 MPa for rectangular and triangular microchannels. Comparing with thermal bonding method, tape assist bonding

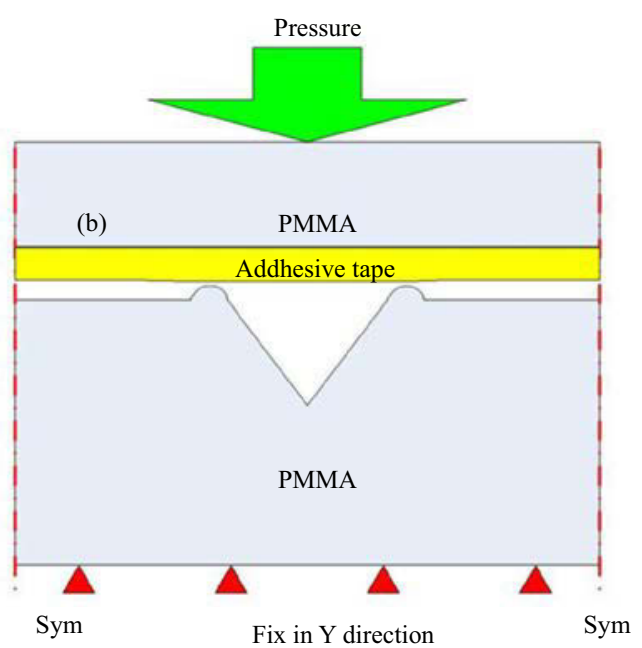

Fig. 6. The model used for adhesive tape assist bonding method 


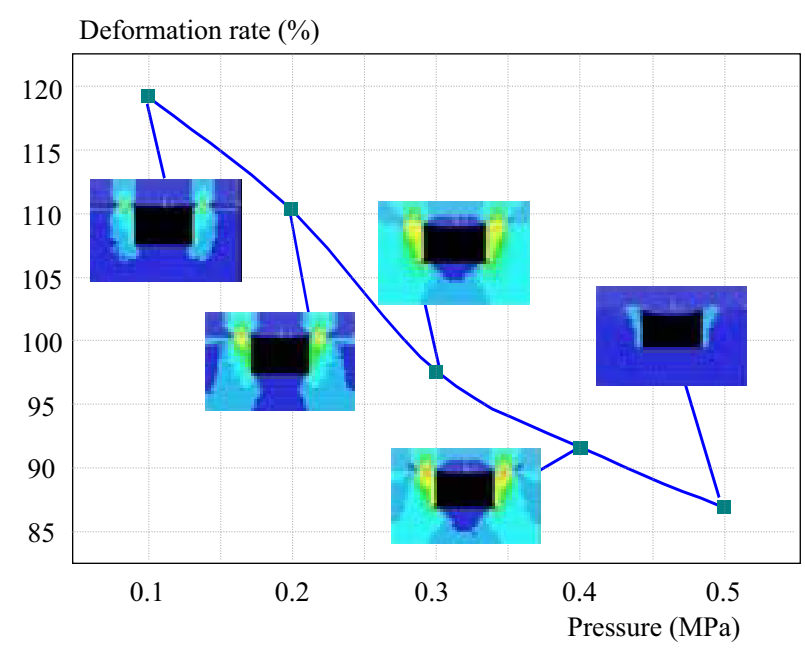

Fig. 7. The influence of the bonding pressure on the deformation of rectangular microchannels (tape assist bonding method)

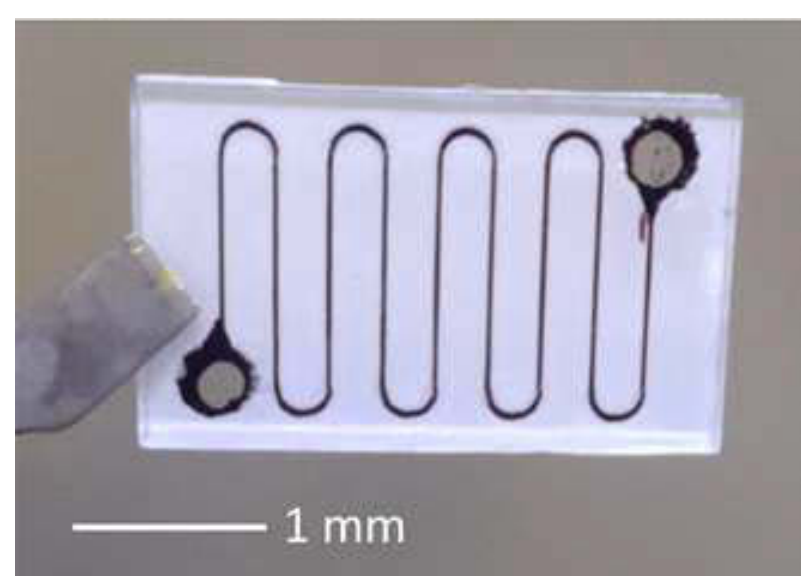

Fig. 9. Dyed water test for microchannels boned by tape assist bonding method

method allows low deformation of the microchannels after bonding. The bonding pressure, bonding temperature and bonding time are lower than those during thermal bonding process. Tape assist bonding method is a room temperature bonding method. It decreases the heating and cooling time which significantly increases the production efficiency. It is suitable for mass bonding of the microfluidic chip.

Figure 9 shows the dyed water test image for bonded microchannels by tape assist bonding method. The dyed water test indicates that the microchannels can be fully bonded without large deformation. Compared with thermal bonding method, the substrate with high roughness can be easily bonded without large microchannels deformation by tape assist bonding method. This method can also allow fast bonding of microchannels which is suitable for mass bonding of microfluidic chips.

Leakage at pressure is one of the most significant problems in microfluidic systems. Therefore, the bonding strength of the chip should be measured. The fabri-

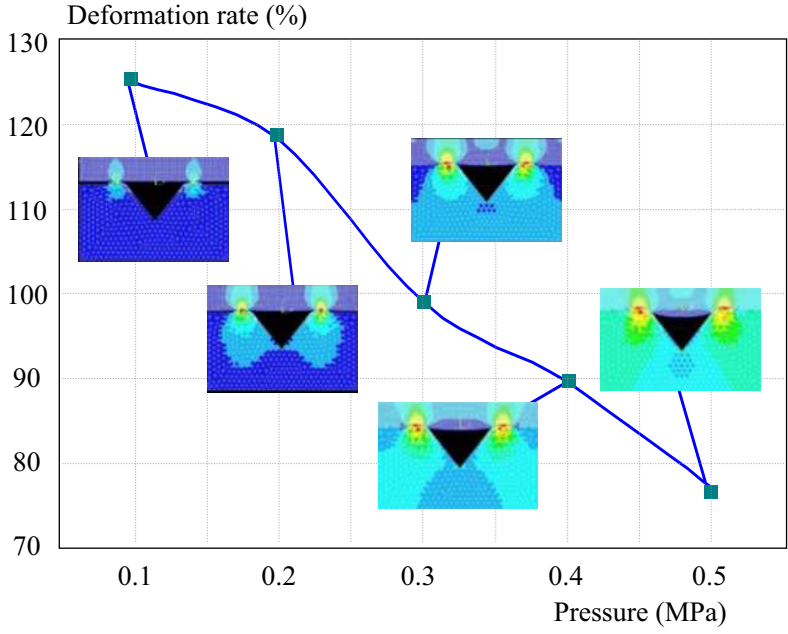

Fig. 8. The influence of the bonding pressure on the deformation of triangular microchannels (tape assist bonding method)

cated microchannel was tested for gas leakage with gas in the Fig. 10 setup. The microchannel $(\approx 300 \mu \mathrm{m}$ wide and $\approx 120 \mu \mathrm{m}$ deep) showed no $\mathrm{N}_{2}$ gas leakage up to $0.84 \mathrm{MPa}$. These results demonstrate that the proposed bonding technique is well suited to the bonding of PMMA microfluidic chips. At the pressure lower than $0.84 \mathrm{MPa}$, the bonded microchannels can stays intact.

\section{Conclusion}

To bond the microchannels at low pressure and temperature, tape assist bonding method was proposed. By this method, the microfluidic chip can be sealed at room temperature without using any equipment. The deformation of the microchannels during tape assist bonding process was analyzed by numerical simulation. The influence of the bonding pressure on the deformation behavior of the microchannel and adhesive tape was studied. The results show that the microchannels can be fully bonded at low bonding pressure. Dyed water test demonstrates there is no blocking or leakage over the entire microchannel. The gas leakage test indicated that the bonded strength of the microfluidic chip can reach to $0.84 \mathrm{MPa}$.

Both CPEs shows exponent $n$ lower than 1 while pure capacitance show exponent $n=1$. Decreasing $\mathrm{n}$ reveals more disordered structure and dispersion of transport processes. The dynamic origin related to multiple trapping systems can be the reason of such behavior. Both, CPE capacitance $\mathrm{P}$ and its exponent $\mathrm{n}$ depends on the applied DC bias. See in Fig. 7-9. Near capacitive behavior ( $\mathrm{n}$ is closed to 1 and voltage independent), as it is clearly recognized in Fig. 9, was observed for second CPE element in $\mathrm{AC}$ equivalent circuit for both samples. The capacity of the structure in forward direction is closely related to the minority charge carriers and thus recombination rate. Second CPE capacitance consistently increases with increasing bias voltages for both samples as one can recognize in Fig. 8. CPE capacitance P1 decreases for 
both BB1 and BB2 samples (Fig. 7) while CPE capacitance P2 increases when DC bias is increased (Fig. 6). The injection of minority carriers results in diffusion (dynamic) CPE capacitance increase at higher forward bias as typical for this type of structure [23]. Similarly, as in the case of capacitances, the two types of resistances are taken into account in the $\mathrm{AC}$ equivalent circuit. In terms of obtained resistance, Fig. 6 shows behavior of both samples. The voltage dependence of resistance indicates two areas dependent on voltage. Two types of resistances are related to shunt and dynamic resistance. The resistance $\mathrm{R} 2$ is ascribed to space charge region of the junction and of course it is dependent on the applied bias. Its value under forward direction is much lower than that one under reverse bias. The higher its value, the better the diode interface [24]. At higher forward voltages the transport process is governed by series resistance.

\section{Conclusion}

To bond the microchannels at low pressure and temperature, tape assist bonding method was proposed. By this method, the microfluidic chip can be sealed at room temperature without using any equipment. The deformation of the microchannels during tape assist bonding process was analyzed by numerical simulation. The influence of the bonding pressure on the deformation behavior of the microchannel and adhesive tape was studied. The results show that the microchannels can be fully bonded at low bonding pressure. Dyed water test demonstrates there is no blocking or leakage over the entire microchannel. The gas leakage test indicated that the bonded strength of the microfluidic chip can reach to $0.84 \mathrm{MPa}$.

\section{Acknowledgements}

This work was supported by the National Basic Research Program of China (973 Program, Grant No. 2015CB057503). The authors would also like to gratitude the Qing Lan Project for its support of the research.

\section{REFERENCES}

[1] X. Zhang, L. Li and C. Luo, "Gel integration for microfluidic applications", Lab on a Chip, vol. 16, no.10, pp. 1757-1776, 2016.

[2] B. C. Lin, "Research and Industrialization of Microfluidic Chip", Chinese Journal of Analytical Chemistry, vol. 44, no.4, pp. 491-499, 2016.

[3] L. Wang, W. Liu, S. Li, T. Liu, X. Yan, Y. Shi, Z. Cheng, C. And and Chen, "Fast fabrication of microfluidic devices using a low-cost prototyping method", Microsystem Technologies-Micro-and Nanosystems-Information Storage and Processing Systems, vol. 22, no.4, pp. 677-686, 2016.

[4] A. Ghobeity, H. J. Crabtree, M. Papini and J. K. Spelt, "Characterisation and comparison of microfluidic chips formed using abrasive jet micromachining and wet etching", Journal of Micromechanics and Microengineering, vol. 22, no.2, pp. 025014, 2012.

[5] R. J. Hu, M. Lei, H. S. Xiong, X. Mu, Y. G. Wang and X. F. Yin, "Highly selective acylation of ferrocene using microfluidic chip reactor", Tetrahedron Letters, vol. 49, no.2, pp. 387-389, 2008.

[6] J. Xu, L. Shi, C. Wang, D. Shan and B. Guo, "Micro hot embossing of micro-array channels ultrafine-grained pure aluminum using a silicon die", Journal of Materials Processing Technology, vol. 225 , pp. 375-384, 2015

[7] G. Fu, S. B. Tor, N. H. Loh, B. Y. Tay and D. E. Hardt, "The demolding of powder injection molded micro-structures: Analysis, simulation and experiment", Journal of Micromechanics and Microengineering, vol. 18, no.7, pp. 2008.

[8] X. Di, K. P. Chen, K. Ohlinger and L. Yuankun, "Nanoimprinting Lithography of a Two-layer Phase mask for Three-dimensional Photonic Structure Holographic Fabrications via Single Exposure", Nanotechnology, vol. 22, no.3, pp. 035303, 2011.

[9] V. Sunkara, D. K. Park and Y. K. Cho, "Versatile method for bonding hard and soft materials", RSC Advances, vol. 2, no.24, pp. 9066-9070, 2012.

10] Y. L. Wu, J. J. Lin, P. Y. Hsu and C. P. Hsu, "Highly sensitive polysilicon wire sensor for DNA detection using silica nanoparticles/gamma-APTES nanocomposite for surface modification", Sensors and Actuators: B Chemical, vol. 155, no.2, pp. 709-715, 2011.

[11] X. Zhu, G. Liu, Y. Guo and Y. Tian, "Study of PMMA thermal bonding", Microsystem Technologies, vol. 13, no.3, pp. 403-407, 2007.

12] Y. Sun, Y. C. Kwok and N. T. Nguyen, "Low-pressure, high-tempq erature thermal bonding of polymeric microfluidic devices and their applications for electrophoretic separation", Journal of Micromechanics and Microengineering, vol. 16, no.8, pp. 1681, 2006

13] Z. Zhang, X. Wang, Y. Luo, S. He and L. Wang, "Thermal assisted ultrasonic bonding method for poly(methyl methacrylate) (PMMA) microfluidic devices", Talanta, vol. 81, no.4, pp. 1331-1338, 2010.

14] S. H. Ng, R. T. Tjeung, Z. F. Wang, A. C. W. Lu, I. Rodriguez, N. F. de and Rooij, "Thermally activated solvent bonding of polymers", Microsystem Technologies-Micro-and Nanosystems-Information Storage and Processing Systems, vol. 14, no.6, pp. $753-759,2008$.

[15] Y. C. Hsu and T. Y. Chen, "Applying Taguchi methods for solvent-assisted PMMA bonding technique for static and dynamic mu-TAS devices", Biomedical Microdevices, vol. 9, no.4, pp. 513-522, 2007.

[16] A. A. Yussuf, I. Sbarski, J. P. Hayes, M. Solomon and N. Tran, "Microwave welding of polymeric-microfluidic devices", Journal of Micromechanics and Microengineering, vol. 15, no.9, pp. 1692-1699, 2005.

[17] R. Chantiwas, M. L. Hupert, S. R. Pullagurla, S. Balamurugan, J. Tamarit, S. Park, P. Datta, J. Goettert, Y. K. Cho and S. A. Soper, "Simple replication methods for producing nanoslits thermoplastics and the transport dynamics of double-stranded DNA through these slits", Lab on a Chip, vol. 10, no.23, pp. 3255-3264, 2010.

18] L. Junshan, Q. Hongchao, L. Chong, X. Zheng, L. Yongqian and W. Liding, "Plasma assisted thermal bonding for PMMA microfluidic chips with integrated metal microelectrodes", Sensors and Actuators: B Chemical, vol. 141, no.2, pp. 646-51, 2009.

[19] H. Zhang, X. Liu and Z. Peng, "Investigation of Thermal Bonding on PMMA Capillary Electrophoresis Chip", vol. 60, pp. 288, 2009.

20] H. Takagi, M. Takahashi, R. Maeda, Y. Onishi, Y. Iriye, T. Iwasaki and Y. Hirai, "Analysis of time dependent polymer deformation based on a viscoelastic model thermal imprint process", Microelectronic Engineering, vol. 85, no.5, pp. 902-906, 2008.

[21] E. Cheng, Z. Yin, H. Zou and P. Jurčíček, "Experimental and numerical study on deformation behavior of polyethylene 
terephthalate two-dimensional nanochannels during hot embossing process", Journal of Micromechanics and Microengineering, vol. 24, no.1, pp. 015004, 2014.

[22] M. L. Williams, R. F. Landel and J. D. Ferry, "Mechanical properties of substances of high molecular weight . 19. the temperature dependence of relaxation mechanisms amorphous polymers and other glass-forming liquids", Journal of the American Chemical Society, vol. 77, no.14, pp. 3701-3707, 1955.

[23] J. J. Aklonis and W. J. MacKnight Introduction to polymer viscoelasticity, Interscience: Wiley, 1983.

[24] R. C. Progelhof, J. L.Throne and R. Progelhof, Polymer engineering principles: properties, processes, and tests for design, Cincinnati: Hanser Gardner, 1993.

[25] P. Nagarajan and D. Yao, "Uniform Shell Patterning Using Rubber-Assisted Hot Embossing Process. II. Process Analysis", Polymer Engineering and Science, vol. 51, no.3, pp. 601-608, 2011.

[26] H. Hocheng and C. C. Nien, "Numerical analysis of effects of mold features and contact friction on cavity filling the nanoimprinting process", Journal of Microlithography Microfabrication and Microsystems, vol. 5, no.1, pp. 011004, 2006.
Received 11 September 2017

Zhifu Yin is a lecturer at Jilin University in China. He obtained PhD degree in Dalian University of Technology, China, in 2016. His research interests lie in the fabrication and application of micro- and nanodevices used for biological, medical and mechanical fields. More than 20 international journal and conference papers have been published based on the above researches.

Helin Zou is a professor at Dalian University of Technology in China. He obtained PhD degree in University of Wales, Cardiff, UK, in 2001, responsible for courses and his research interests lie in the application of microfabrication technology to the development of micro-engineered systems aimed at providing an information-gathering front end to electronic system. More than 50 international journal and conference papers have been published based on the above researches. He is member of the British Institute of Physics and Micro/Nano society of China. 